\title{
On Maximal Surfaces in Asymptotically Flat Space-Times
}

\author{
R. Bartnik ${ }^{1}$, P. T. Chruściel ${ }^{2 \star \star \star}$ and N. Ō Murchadha ${ }^{3}$ \\ ${ }^{1}$ Centre for Mathematical Analysis, Australian National University, Canberra ACT 2601, Australia \\ ${ }^{2}$ Yale University, Department of Physics, 217 Prospect Street, New Haven CT 06511, USA \\ ${ }^{3}$ University College, Physics Department, Cork, Ireland
}

\begin{abstract}
Existence of maximal and "almost maximal" hypersurfaces in asymptotically flat space-times is established under boundary conditions weaker than those considered previously. We show in particular that every vacuum evolution of asymptotically flat data for the Einstein equations can be foliated by slices maximal outside a spatially compact set and that every (strictly) stationary asymptotically flat space-time can be foliated by maximal hypersurfaces. Amongst other uniqueness results, we show that maximal hypersurfaces can be used to "partially fix" an asymptotic Poincaré group.
\end{abstract}

\section{Introduction}

The significant role played by maximal hypersurfaces in general relativity is well known and hardly needs to be discussed (cf. e.g. [An, CBY, COM]). A few years ago one of us [Ba1] established existence of such hypersurfaces under some interior regularity conditions together with some rather strong asymptotic conditions on the metric. In [Ba1] it was assumed that the metric tends to the flat metric as $r^{-1}$ and the trace of the extrinsic curvature of the $t=$ const slices falls off at least as $r^{-3}$. In a recent analysis of solutions of the Einstein equations in which one can make global Lorentz transformations (the boost theorem) [COM] it was shown that for any $\alpha>0$ there exists many regular solutions to the vacuum field equations for which the metric only falls off to the flat metric as $r^{-\alpha}$ and the extrinsic curvature as $r^{-1-\alpha}$. For such solutions, we cannot a priori expect the trace of the extrinsic curvature $K$ to fall off faster than $r^{-1-\alpha}$. In this paper we show that in some situations even with such a slow fall-off the existence of maximal slices can be established, provided $\alpha>1 / 2$. It may be of some interest to note that this decay condition covers, roughly speaking, all cases in which the mass has been shown to be finite and well defined ([Ba3, Ch2, OM $]$ ).

It has been recently observed $[\mathrm{Br}, \mathrm{Wi}]$ that there exists a topological obstruction

* On leave of absence from the Institute of Mathematics, Polish Academy of Sciences, Warsaw, Poland

$\star \star$ Supported in part by the NSF grant PHY 8503072 to Yale University 
to the existence of maximal slices in Lorentzian manifolds. In some situations it may be useful to consider "almost maximal" slices, i.e. slices on which the trace of the extrinsic curvature has compact support - this is the best one can hope to get in "bad" topological situations". Our main result - Theorem 3.5-is as follows: there always exists an evolution of asymptotically flat Cauchy data for the vacuum Einstein equations which contains a foliation by "almost maximal" slices. Under stronger asymptotic conditions, a similar result was shown in the thesis of one of the authors. An idea similar to the one we use has also been considered by Ashtekar [As]. In Sect. 4 we show existence of maximal slices under weaker conditions than previously considered in [Ba1]. It must be recognized that our result is not as good as one would wish: we still have to impose a restriction on the rate of decay of the metric together with a condition on the time-variation of the trace of the extrinsic curvature $K$ to prove our assertion. However, it is not clear whether our decay conditions can be weakened further. We also show the existence of maximal slicings of strictly stationary asymptotically flat space-times. In Sect. 5 some results concerning the uniqueness of asymptotically flat maximal foliations are established, assuming the time-like convergence condition. An interesting result for physical applications is Theorem 5.5, in which we show that maximal slicings can be used to kill the "supertranslations in time."

\section{Preliminaries}

Let us first fix notations - the signature is $(-+++\cdots)$, greek indices run from 0 to $n-1$, where $n$ is the dimension of the spacetime (we shall assume $n \geqq 4$ throughout to avoid a physically irrelevant discussion of the asymptotic behaviour of solutions of the Laplace equation in space dimension equal to two), latin indices run from 1 to $n-1,|\cdot|$ denotes the absolute value if the entry $\cdot$ is a number, while $|x|=\left(\Sigma\left(x^{\mu}\right)^{2}\right)^{1 / 2}$ when $x$ is a vector. $B(x, r), S(x, r)$, and $E(x, r)=\mathbb{R}^{m}-\overline{B(x, r)}$ denote an open coordinate ball, a sphere, and the interior of the complement of a ball respectively (in dimension $m$ ) with centre $x$ and radius $r$. We shall write $B(r$ ), etc. whenever $x=0$; if ambiguities are likely to occur we shall write $\mathscr{B}(r)$ to denote a space-time ball $\mathscr{B}(r)=\left\{x^{\mu}:|x|<r\right\}$ to distinguish it from the space ball $B(r)=$ $\left\{x^{i}: r(x)<r\right\}$, where

$$
r(x)=\left(\Sigma\left(x^{i}\right)^{2}\right)^{1 / 2}
$$

The letters $x, y, \ldots$ have been used to denote both spatial and full space-time coordinate points. In places where the usage is not easily inferred from the context, the alternative notation $\left(x^{i}\right),\left(x^{\mu}\right)$ for spatial, respectively space-time, points has been preferred.

For $\lambda \in(0,1), \beta \in \mathbb{R}, k \in \mathbb{N} \cup\{0\}$, and $\Omega$ a domain in $\mathbb{R}^{1, n-1}$ we define

$$
\begin{aligned}
\|f\|_{C_{\beta}^{k, \lambda}(\Omega)}= & \sup _{\left(x^{\mu}\right) \in \Omega}\left\{\sum_{|\alpha| \leqq k}\left(\left|D^{\alpha} f\right| \sigma^{-\beta+|\alpha|}\right)(x)\right. \\
& \left.+\sup _{\substack{y \in \mathscr{P}(x, \sigma(x) / 2) \cap \Omega \\
y^{0}=x^{0}}}\left\{\sum_{|\alpha|=k} \frac{\left|D^{\alpha} f(x)-D^{\alpha} f(y)\right|}{r(x-y)^{\lambda}} \sigma(x)^{-\beta+k+\lambda}\right\}\right\} .
\end{aligned}
$$

${ }^{1}$ It is worth noting that such "bad topology" is necessarily hidden behind an apparent horizon [SY, Ba4] 


$$
\begin{aligned}
\|f\|_{\mathcal{L}_{\beta}^{k, \lambda}(\Omega)}= & \sup _{\left(x^{\mu}\right) \in \Omega}\left\{\sum_{|\alpha| \leqq k}\left(\left|\mathscr{D}^{\alpha} f\right| \sigma^{-\beta+|\alpha|}\right)(x)\right. \\
& \left.+\sup _{\left(y^{\mu}\right) \in \mathscr{D}(x, \sigma(x) / 2) \cap \Omega}\left\{\sum_{|\alpha|=k} \frac{\left|\mathscr{D}^{\alpha} f(x)-\mathscr{D}^{\alpha} f(y)\right|}{|x-y|^{\lambda}} \sigma(x)^{-\beta+k+\lambda}\right\}\right\},
\end{aligned}
$$

where $\mathscr{D}$ stands for space and time derivatives while $D$ stands for spatial derivatives only, with $\sigma(x)=\left(1+r(x)^{2}\right)^{1 / 2}$. We shall also write

$$
\Delta_{0}=\Sigma\left(\partial / \partial x^{i}\right)^{2}, \quad\|\nabla f\|=\left|g^{\mu v} f_{, \mu} f_{, v}\right|^{1 / 2}
$$

(in particular $\|\nabla f\|$ is non-negative irrespective of whether $\nabla f$ is time-like or spacelike). The letters $t, x^{0}$, etc., will always denote functions whose level sets are complete Riemannian manifolds (with or without boundary). The following proposition will be used throughout:

Proposition 2.1 ([Me]). For every $\rho \in C_{-\beta}^{k, \lambda}(\Omega), \Omega=\mathbb{R}^{n}-B(R), n \geqq 3, R \geqq 1, k \in \mathbb{N} \cup\{0\}$, $\lambda \in(0,1), \beta \in(1,2]$, there exists a solution of the equation

$$
\Delta_{0} u=\rho
$$

with the following properties:

1. If $1<\beta<2, u \in C_{2-\beta}^{k+2, \lambda}(\Omega)$ and

$$
\|u\|_{C_{2-\beta}^{k+2, \lambda}} \leqq C(k, \lambda, \beta, n)\|\rho\|_{C_{-\beta}^{k, \lambda}}
$$

2. If $\beta=2,(1+\ln r)^{-1} u \in C_{0}^{k+2, \lambda}(\Omega)$ and

$$
\left\|(1+\ln r)^{-1} u\right\|_{C^{k+2, \lambda}(\Omega)} \leqq C(k, \lambda, n)\|\rho\|_{C_{-2}^{k, \lambda}} .
$$

Lemma 2.2. Let $g_{\mu \nu}-\eta_{\mu \nu} \in \mathscr{C}_{-\alpha}^{m, \lambda}(\Omega), \alpha>0, m \geqq 2$ and let $K_{0} \in \mathscr{C}_{-1-\alpha}^{m-1, \lambda}(\Omega) \cap C_{-1-\gamma}^{0, \lambda}(\Omega)$ be the trace of the extrinsic curvature of the (space-like) slices $t=$ constant, $\gamma \geqq \alpha$. Let $\tau=t+u, u \in C_{1-\alpha}^{m+1, \lambda}(\Omega) \cap C_{1-\gamma}^{2, \lambda}(\Omega), \partial_{t} u=0$, be such that $\nabla \tau$ is time-like, and define $\Omega^{\prime}=\left\{\left(\tau, x^{i}\right):\left(\tau-u, x^{i}\right) \in \Omega\right\}$. We have

$$
\begin{gathered}
\|\nabla \tau\| K(t, x)=\left(\|\nabla t\| K_{0}\right)(\tau-u, x)-\Delta_{0} u+\kappa, \\
\kappa \in \mathscr{C}_{-1-2 \alpha}^{m-1, \lambda^{2}}\left(\Omega^{\prime}\right) \cap C_{-1-\alpha-\gamma}^{0, \lambda^{2}}\left(\Omega^{\prime}\right),
\end{gathered}
$$

where $K$ is the trace of the extrinsic curvature of the $\tau=$ constant slices.

Proof. We have

$$
\begin{aligned}
& K=-\nabla_{\mu}\left(\nabla^{\mu} \tau /\|\nabla \tau\|\right)=-\|\nabla \tau\|^{-1}\left(g^{\mu v}+\frac{\nabla^{\mu} \tau \nabla^{v} \tau}{\|\nabla \tau\|^{2}}\right) \tau_{; \mu v}=-\|\nabla \tau\|^{-1}(A+B+C) \\
& A=\left(g^{\mu v}+\frac{\nabla^{\mu} t \nabla^{v} t}{\|\nabla t\|^{2}}\right) t_{; \mu v}=-\|\nabla t\| K_{0} \\
& B=\left(\frac{\nabla^{\mu} \tau \nabla \tau}{\|\nabla \tau\|^{2}}-\frac{\nabla^{\mu} t \nabla^{v} t}{\|\nabla t\|^{2}}\right) t_{; \mu v}, \\
& C=\left(g^{\mu v}+\frac{\nabla^{\mu} \tau \nabla^{v} \tau}{\|\nabla \tau\|^{2}}\right) u_{; \mu v}=\Delta_{0} u+D .
\end{aligned}
$$

Straightforward, but somewhat tedious, computation shows the desired result. 
Lemma 2.3. Let $0<\alpha \leqq \gamma<1$, let $\Omega \supset \Omega_{0} \equiv\left\{(t, x) ; r \geqq R,|t| \leqq C_{1} r^{1-\gamma}+C_{2}\right\}, C_{1} \in \mathbb{R}^{+}$, $C_{2} \in \mathbb{R}, \quad C_{1} R^{1-\gamma}+C_{2} \geqq 0, \quad C_{1}$ sufficiently large or let $\Omega \supset \Omega_{0} \equiv\{(t, x), \quad r \geqq R$, $\left.|t| \leqq C_{1} r^{1-\gamma+\varepsilon}+C_{2}\right\}, C_{1}, \varepsilon>0, C_{2} \in \mathbb{R}, C_{1} R^{1-\gamma+\varepsilon}+C_{2} \geqq 0$. Let $g_{\mu v}, K_{0}$ and $K$ be as in Lemma 2.2. Then there exists new coordinates $(\tau, x) \in \Omega^{\prime}$ such that

1. $\Omega^{\prime} \supset \Omega_{0}^{\prime} \equiv\left\{(\tau, x), r \geqq R,|\tau| \leqq C_{1}^{\prime} r^{1-\gamma}+C_{2}^{\prime}\right\}$ for some $C_{1}^{\prime} \geqq 0, C_{1}^{\prime} R^{1-\gamma}+C_{2}^{\prime}=$ $C_{1} R^{1-\gamma}+C_{2}$ or $C_{1}^{\prime} R^{1-\gamma}+C_{2}^{\prime}=C_{1} R^{1-\gamma+\varepsilon}+C_{2}$;

2. The pulled-back metric $g_{\mu \nu}^{\prime}$ satisfies

$$
g_{\mu \nu}^{\prime}-\eta_{\mu \nu} \in \mathscr{C}_{-\alpha}^{m, \lambda^{2}}\left(\Omega^{\prime}\right)
$$

3. $\|\nabla \tau\| K \in \mathscr{C}_{-1-\alpha}^{m-1, \lambda^{2}}\left(\Omega^{\prime}\right) \cap C_{-1-\alpha-\gamma}^{0, \lambda^{2}}\left(\Omega^{\prime}\right)$;

4. $C_{1}^{\prime}, C_{2}^{\prime}$ and the appropriate norms of $g_{\mu \nu}^{\prime}$ and $\|\nabla \tau\| K$ depend only upon $\alpha, \gamma,\|g-\eta\|$, $\left\|K_{0}\right\|, R, \sup \|\nabla t\|^{-1}(x), C_{1}, C_{2}, n, m$ and $\varepsilon$.

5. If $\alpha>1 / 2$ and if there exist constants $C_{3}, \varepsilon^{\prime} \geqq 0$ such that

$$
\forall(t, x) \in \Omega_{0}, \quad\left|\left(\|\nabla t\| K_{0}\right)(t, x)-\left(\|\nabla t\| K_{0}\right)(0, x)\right| \leqq C_{3} r^{-2-\varepsilon^{\prime}},
$$

then for $(\tau, x) \in \Omega_{0}^{\prime}$

$$
|(\|\nabla \tau\| K)(\tau, x)-(\mid \nabla \tau \| K)(0, x)| \leqq C_{3}^{\prime} r^{-2-\varepsilon^{\prime \prime}},
$$

$\varepsilon^{\prime \prime}=\min (\varepsilon, 2 \alpha-1)$ for some $C_{3}^{\prime} \geqq 0$. The constants $C_{3}^{\prime}, C_{1}^{\prime}, C_{2}^{\prime}-C_{2},\left\|g_{\mu \nu}^{\prime}-\eta_{\mu \nu}\right\|$, \|\|$\nabla \tau\|K\|$ depend only on $\alpha, \gamma, n, m, \lambda, \sup _{x \in \Omega}\|\nabla t\|^{-1}(x),\left\|g_{\mu \nu}-\eta_{\mu \nu}\right\|,\|\| \nabla t\left\|K_{0}\right\|$, $R, C_{3}, C_{1}$ and $\varepsilon$; in particular, they are independent of $C_{2}$.

Proof. By Proposition 2.1 there exists a solution $\hat{u} \in C_{1-\alpha}^{m+1, \lambda}(E(R)) \cap C_{1-\gamma}^{2, \lambda}(E(R))$ of the equation

$$
\Delta_{0} \hat{u}\left(x^{i}\right)=\left(\|\nabla t\| K_{0}\right)\left(t=0, x^{i}\right) .
$$

Let $\phi_{R}(x)=\phi(r(x) / R)$, with some function $\phi$ satisfying $0 \leqq \phi \leqq 1, \phi(s)=1$ for $s>1$, $\phi(s)=0$ for $s<1 / 2, \phi \in C^{\infty}(\mathbb{R})$. Let $C=\sup _{r \geqq \max (1, R)} \hat{u} r^{\gamma-1}$. If $\Omega \supset \Omega_{0} \equiv\{r \geqq R,|t| \leqq$ $\left.C_{1} r^{1-\gamma}+C_{2}\right\}$ and $C_{1}>C$ let $C_{1}^{\prime}=\left(C_{1}-C_{2}\right) / 2, C_{2}^{\prime}=\left(C_{1}-C_{1}^{\prime}\right) R^{1-\gamma}+C_{2}$, if $\Omega \supset \Omega_{0} \equiv$ $\left\{r \geqq R,|t| \leqq C_{1} r^{1-\gamma+\varepsilon}+C_{2}\right\}$ let $C_{1}^{\prime}=C_{1}, C_{2}^{\prime}=C_{1}\left(R^{\varepsilon}-1\right)+C_{2}$. A simple calculation shows that we can choose $R_{0} \geqq \max (1, R)$ such that

(a) the level surfaces of $\tau=t+\phi_{R_{0}} \hat{u}$ are space-like;

(b) $\sup \|\nabla \tau\|^{-1} \leqq 2 \sup \|\nabla t\|^{-1}$;

(c) for all $r \geqq R$ and $\tau \leqq C_{1}^{\prime} r^{1-\gamma}+C_{2}^{\prime}$ both $(\tau-u, x)$ and $(\tau, x)$ belong to $\Omega_{0}$.

The new coordinates $\left(t^{\prime}, x^{\prime}\right)$ are defined by $t^{\prime}=\tau, x^{i}=x^{i}$. Proposition 2.1 gives

$$
\|\hat{u}\|_{C_{1-\alpha}^{m+1, \lambda}} \leqq C\left\|\left(\|\nabla t\| K_{0}\right)(0, x)\right\|_{C_{-1-\alpha}^{m-1, \lambda}(E(R))},
$$

which implies uniform estimates for $g_{\mu \nu}^{\prime}-\eta_{\mu \nu}$ in $\mathscr{C}_{-\alpha}^{m, \lambda^{2}}\left(\Omega^{\prime}\right)$, therefore, in particular, uniform estimates for $K$ in $\mathscr{C}_{-1-\alpha}^{m-1, \lambda^{2}}\left(\Omega^{\prime}\right)$. Let $\phi_{0}(t, x)=\left(\|\nabla t\| K_{0}\right)(t, x)$ and $\phi(\tau, x)=$ $(\|\nabla \tau\| K)(\tau, x)$. Lemma 2.2 gives

$$
\begin{aligned}
\phi(\tau, x) & =\phi_{0}(\tau-u, x)-\phi_{0}(0, x)+\kappa \\
& =-\int_{0}^{1} u\left[\frac{\partial \phi_{0}}{\partial t}(\tau-s u, x)\right] d s+\phi_{0}(\tau, x)-\phi_{0}(0, x)+\kappa
\end{aligned}
$$


The uniform bounds on $\hat{u}$ and on $\left\|\partial \phi_{0} / \partial t\right\|_{C_{-2-\alpha}^{0, \lambda}}$ imply a bound on

$$
\left\|\int_{0}^{1} d / d s\left[\phi_{0}(t-s u, x)\right] d s\right\|_{C_{-1-\alpha-\gamma}^{0, \lambda^{2}},},
$$

and therefore a bound on

$$
\|K(0, \cdot)\|_{C_{1-\alpha-\gamma}^{0, \lambda^{2}}(E(R))} \cdot
$$

For $|\tau| \leqq C_{1}^{\prime} r^{1-\gamma}+C_{2}^{\prime}$ we have

$$
\begin{aligned}
& \phi_{0}(\tau, x)-\phi_{0}(0, x)=\int_{0}^{\tau} \frac{\partial \phi_{0}}{\partial t}(t, x) d t \\
& \leqq\left\|\frac{\partial \phi_{0}}{\partial t}\right\|_{C_{-2-\alpha}^{0}}\left(C_{1}^{\prime}+C_{2}^{\prime} R^{\gamma-1}\right) r^{-1-\gamma-\alpha},
\end{aligned}
$$

the Hölder continuity of space-derivatives is established via a similar manipulation. If (2.3) holds (2.4) follows directly from (2.5).

\section{Remarks.}

1. If $\alpha=\gamma=1$, Lemma 2.3 holds with the following modifications:

a) $C_{1} r^{1-\gamma}$ should be replaced by $C_{1} \ln (1+r)$.

b) $C_{1}^{\prime}=\left\{C_{1}-\sup \left[(1+\ln (1+r))^{-1} \hat{u}\right]\right\} / 2$,

c) $(1+\ln (1+r))^{-1}\left(g_{\mu \nu}^{\prime}-\eta_{\mu \nu}\right) \in \mathscr{C}_{-1}^{m, \lambda^{2}}\left(\Omega^{\prime}\right)$

d) $(1+\ln (1+r))^{-1}\|\nabla \tau\| K \in \mathscr{C}_{-2}^{m-1, \lambda^{2}}\left(\Omega^{\prime}\right) \cap C_{-3}^{0, \lambda^{2}}\left(\Omega^{\prime}\right)$.

2. If $\alpha>1 / 2,(2.5)$ shows that (2.3) holds with a constant $C_{3}$ depending also on $C_{2}$. We are unable to use Theorem 5.3 of $[\mathrm{Ba} 1]$ to infer the existence of an entire maximal slice without some further hypotheses because $C_{3}$ increases as we try to increase $C_{2}$. This is the only place where $C_{2}$ enters our estimates.

\section{Slices Maximal Outside a Compact Set}

Theorem 3.1. Let $g_{\mu v}-\eta_{\mu \nu} \in \mathscr{C}_{-\alpha}^{m, \lambda}(\Omega), \quad 0<\alpha<1, \quad m \geqq 2, \quad \Omega \supset \Omega_{0} \equiv\{r \geqq R,|t| \leqq$ $\left.C_{1} r^{1-\alpha}+C_{2}\right\}, C_{1} R^{1-\alpha}+C_{2} \geqq 0, C_{1} \geqq 0$. If $C_{1}$ is large enough then there exists $a$ space-like slice $\Sigma \subset \Omega$ given by $\Sigma=\{(t, x), t=u(x)\}$ and a positive constant $R_{0}\left(\left\|g_{\mu \nu}-\eta_{\mu \nu}\right\|, \sup \|\nabla t\|^{-1}, \alpha, \lambda, C_{1}, C_{2}\right)$ such that

$$
\forall x: r(x) \geqq R_{0}, \quad K_{\Sigma}(x)=0,
$$

where $K_{\Sigma}$ is the trace of the extrinsic curvature of $\Sigma$, and $u \in C_{1-\alpha}^{m+1, \lambda^{\prime}}\left(\Sigma_{0}\right)$ for some $\lambda^{\prime}>0$, where $\Sigma_{0}=\{(t, x) \in \Omega, t=0\}$.

Proof. Iterating Lemma $2.3 k=\left[\alpha^{-1}\right]$ times, where $\left[\alpha^{-1}\right]$ stands for the largest integer smaller than $\alpha^{-1}$, we obtain a coordinate system $\left(t^{\prime}, x^{\prime}\right)$ in which $\left|K^{\prime}\right| \leqq$ $\mathrm{Cr}^{-2-\varepsilon}\left(K^{\prime}\right.$ is the trace of the extrinsic curvature of the $t^{\prime}=$ constant slices), $\varepsilon=(k+1) \alpha-1>0^{2}$ and which covers a set $\Omega^{\prime} \supset \Omega_{0}^{\prime}=\left\{r(x) \geqq R,\left|t^{\prime}\right| \leqq C_{1}^{\prime} r^{\varepsilon^{\prime}}+C_{2}^{\prime}\right\}$,

\footnotetext{
${ }^{2}$ If $(k+1) \alpha=1$ reduce $\alpha$ to $\alpha / 2$ in the $k^{\text {th }}$ iteration, and iterate once more
} 
$\varepsilon^{\prime}=1-k \alpha>0$, with $g_{\mu \nu}^{\prime}-\eta_{\mu \nu} \in \mathscr{C}_{-\alpha}^{m, \lambda^{\prime}}\left(\Omega^{\prime}\right)$. There exists $C^{\prime}, R^{\prime}$ large enough such that the functions $\omega_{ \pm}= \pm C^{\prime} r^{-\varepsilon}$ form space-like barriers for the maximal hypersurface equation for $r \geqq R^{\prime}$. A standard method and Bartnik's $C^{1}$ estimates (cf. [Ba1, Ba2]) imply the existence of a solution to the problem

$\left.\tilde{u}\right|_{S\left(R^{\prime}\right)}=0$

$\tilde{u} \rightarrow 0$ as $r \rightarrow \infty$;

the trace of the extrinsic curvature of the space-like surface $t^{\prime}=\tilde{u}\left(x^{\prime}\right)$ vanishes.

Let $\phi \in C^{\infty}([0, \infty])$ satisfy $\phi(s)=0$ for $s \leqq 1 / 2, \phi(s)=1$ for $s \geqq 1,0 \leqq \phi \leqq 1$. $\Sigma$ is defined by the equation $t^{\prime}=\phi\left[\left(r\left(x^{\prime}\right)\right) / 4 R^{\prime}\right] \tilde{u}\left(x^{\prime}\right)$.

A method similar to that used to prove Theorem 3.1 can be used to prove Theorem 3.2:

Theorem 3.2. Let $g_{\mu \nu}-\eta_{\mu \nu} \in \mathscr{C}_{-1}^{m, \lambda}(\Omega), m \geqq 2, \Omega \supset \Omega_{0} \equiv\left\{r \geqq R,|t| \leqq C_{1} \ln (1+r)+C_{2}\right\}$, $C_{1}>0, C_{2} \in R, C_{1} \ln (1+R)+C_{2} \geqq 0$. If $C_{1}$ is large enough, the conclusion of Theorem 3.1 holds with $(1+\ln (1+r))^{-1} u \in C_{0}^{m+1, \lambda^{\prime}}\left(\Sigma_{0}\right)$.

Proposition 3.3. Let the hypotheses of Theorem 3.1 hold and let the metric satisfy the time-like convergence condition $\left(R_{\mu v} n^{\mu} n^{v} \geqq 0\right.$ for every time-like vector $\left.n^{\mu}\right)$. There exists a neighborhood $\Omega_{1} \subset \Omega_{0}$ of the almost maximal slice $\Sigma$ together with a foliation of $\Omega_{1}$ by space-like hypersurfaces $\tilde{\Sigma}_{\tau}, \tau \in\left(-C_{1} R^{1-\gamma}-C_{2}, C_{1} R^{1-\gamma}+C_{2}\right), \tau(x)=t(x)$ for $r(x) \leqq R_{1}$ for some $R_{1}, \widetilde{\Sigma}_{0}=\Sigma$, such that (3.1) holds for each $\tilde{\Sigma}_{\tau}$. There also exist coordinates $x^{\prime \prime}$ in $\Omega$, such that $x^{\prime \prime 0}=\tau$ and $g_{\mu \nu}^{\prime \prime}-\eta_{\mu \nu} \in \mathscr{C}_{\alpha}^{m, \lambda^{\prime}}\left(\Omega_{1}\right)$ for some $\lambda^{\prime}>0$.

Proof. The proof is a straightforward generalization of the methods of [CB] as in Sect. 5 of [Ba2], we shall present it here for completeness. Consider the mapping

$$
X \times Y \equiv[-\varepsilon, \varepsilon] \times \stackrel{\circ}{C}_{-\varepsilon}^{3, \lambda}\left(E\left(R_{0}\right)\right) \ni(t, y) \rightarrow K_{t} \in C_{-2-\varepsilon}^{1, \lambda}\left(E\left(R_{0}\right)\right)=Z,
$$

where $K_{t}$ is the mean extrinsic curvature of the slices $t+u=$ const, and $\dot{C}_{-\varepsilon}^{\kappa, \lambda}=$ $\left\{u \in C_{-\varepsilon}^{\kappa, \lambda}:\left.u\right|_{S\left(R_{0}\right)}=0\right\} . K_{t}$ is a differentiable mapping from $X \times Y$ to $Z$ in a neighborhood of $(0,0)$ with derivative $D_{Y} K_{t}$ given by (cf. e.g. [Ba2] Sect. 5),

$$
D_{Y} K(\delta u)=-\Delta(v \delta u)+\left(|A|^{2}+\operatorname{Ric}(N, N)\right) v \delta u,
$$

where $\Delta$ is the induced Laplacian on the hypersurface $t+u=$ const, $|A|$ is the length of the extrinsic curvature tensor, $v$ is the "tilt function," $v=-\langle N, T\rangle$, and $N, T$ are unit normals to the $t+u$ and $t$ slices, respectively. It follows from (e.g. [CSCB]) that $D_{Y} K$ is an isomorphism, and the implicit function theorem implies the existence of a differentiable mapping $\left(-\varepsilon_{0}, \varepsilon_{0}\right) \ni t \rightarrow u_{t} \in C_{-\varepsilon}^{3, \lambda}$ such that $K_{t}\left(t, u_{t}\right)=0$. Let $\tau(t, x)=t+u$. Differentiability of $t \mapsto u_{t}$ as a mapping from $X$ to $Y$ in appropriate topologies implies that $(\partial u / \partial t) \rightarrow 0$ as $r \rightarrow \infty$, therefore $\partial \tau / \partial t$ satisfies the equation

$$
\Delta\left(v_{t} \frac{\partial \tau}{\partial t}\right)-\left(|A|^{2}+\operatorname{Ric}(N, N)\right) v_{t} \frac{\partial \tau}{\partial t}=0
$$

with $(\partial \tau / \partial t) v_{t} \geqq 1$ on $S\left(R_{0}\right)$, and $(\partial \tau / \partial t) v_{t} \rightarrow 1$ as $r \rightarrow \infty$. The Hopf maximum principle [GT, Theorem 3.5] yields $(\partial \tau / \partial t) v_{t} \geqq C>0$ for some constant $C$, therefore $(\partial \tau / \partial t)>0$ and the slices $t+u_{t}=$ const. form a foliation. The possibility of extending the slicing 
from $(-\varepsilon, \varepsilon)$ to the claimed range of $\tau$ follows from the barriers of the proof of Theorem 3.1 and the a priori estimates of [Ba1].

Proposition 3.4. Let the hypotheses of Theorem 3.2 hold and let the metric satisfy the time-like convergence condition. There exists a neighborhood $\Omega_{1} \subset \Omega_{0}$ of the slice $\Sigma$ together with a foliation of $\Omega_{1}$ by space-like hypersurfaces $\tilde{\Sigma}_{\tau}, t \in\left(-C_{1} \ln (1+R)-C_{2}\right.$, $\left.C_{1} \ln (1+r)+C_{2}\right), \tau(x)=t(x)$ for $r(x) \leqq R_{1}$, for some $R_{1}, \tilde{\Sigma}_{0}=\Sigma$ such that (3.1) holds for each $\widetilde{\Sigma}_{\tau}$. There also exist coordinates $x^{\prime \mu}$ in $\Omega$ such that $x^{\prime \prime 0}=\tau$ and $(1+\ln (1+r))^{-1}\left(g_{\mu \nu}^{\prime \prime}-\eta_{\mu \nu}\right) \in \mathscr{C}_{-1}^{m, \lambda^{\prime}}\left(\Omega_{1}\right)$ for some $\lambda^{\prime}>0$.

Remark. It is clear from the proofs of Proposition 3.3 and 3.4 that given any interval $[-T, T]$ there exists an almost maximal foliation by asymptotically flat hypersurfaces asymptotic to the slices $t=$ const. for $t \in[-T, T]$. The size $R_{0}$ of the set on which $K \neq 0$ may in general depend upon $T$.

In the following theorem the notation of [COM] will be used.

Theorem 3.5. Let $\left(\Sigma, g_{i j}, K^{i j}\right)$ be an $(s, \delta), s \in \mathbb{N}, \delta>-3 / 2$ asymptotically flat initial data set for the vacuum Einstein equations, i.e.

1. $\Sigma$ is a connected complete Riemannian three-dimensional manifold of the form $\Sigma=\Sigma_{\mathrm{int}} \bigcup_{i=1}^{I} \Sigma_{i}, \Sigma_{\mathrm{int}}$ - compact manifold with boundary, $\Sigma_{i} \approx \mathbb{R}^{3}-B\left(R_{i}\right)$.

2. $g \in H_{s}\left(\Sigma_{\text {int }}\right), K \in H_{s-1}\left(\Sigma_{\text {int }}\right)$ and in local coordinates $g_{i j}-\delta_{i j} \in H_{s, \delta}\left(\mathbb{R}^{3}-B\left(R_{i}\right)\right)$, $K^{i j} \in H_{s-1, \delta+1}\left(\mathbb{R}^{3}-B\left(R_{i}\right)\right)$ in each asymptotic end $\Sigma_{i}$.

If $s \geqq 5$, there exists an evolution. $\left(M, \gamma_{\mu v}\right)$ of $(\Sigma, g, K)$ satisfying the vacuum Einstein equations and an open subset $\Omega$ of $M$ foliated by almost maximal $(s-1 / 2, \delta)$ asymptotically flat slices $\Sigma_{\tau}, \Sigma_{\tau}$ diffeomorphic to $\Sigma$.

\section{Remarks.}

1. The asymptotic conditions of Theorem 3.5 will be satisfied if, for example $g_{i j}-\delta_{i j} \in C_{-\alpha}^{5}, \alpha>\delta+3 / 2, K^{i j} \in C_{-\alpha-1}^{4}$.

2. The metric induced on the almost maximal slices will be $C_{-\alpha}^{2, \lambda}, \alpha=\delta+1>0$ (cf. the sharp embedding theorems of [Ba3]).

3. We believe that the almost maximal slices will be $(s, \delta)$ asymptotically flat.

Proof. A straightforward extension of the boost theorem [COM] shows that in each asymptotic end there exists an evolution of the data $(g, K)$ in a set $\Omega_{i}$, $\Omega_{i} \supset\left\{r \geqq R_{i},|t| \leqq \varepsilon_{i}(1+r)\right\}$. The standard short time existence theorem [CBY] shows the existence of an evolution of the Cauchy data in a set $\left(-\varepsilon_{0}, \varepsilon_{0}\right) \times \Sigma_{\text {int }}$. Let $\varepsilon=\min _{0 \leqq i \leqq I} \varepsilon_{i}$. Proposition 3.3 implies the existence of the required foliation $\Sigma_{\tau}$, $\tau \in(-\varepsilon, \varepsilon)$. By construction the slices $\Sigma_{\tau}$ will be graphs over $\Sigma$ of $H_{s+1, \delta-1}$ functions, which together with restriction theorems (cf. e.g. [COM]) yields $(s-1 / 2, \delta)$ asymptotic flatness of the $\Sigma_{\tau}$ foliation.

\section{Maximal Slices}

Theorem 4.1. Let $V$ be an $n$-dimensional Lorentzian manifold, $n \geqq 4$, such that $V=V_{\mathrm{int}} \cup V_{\mathrm{ext}}$, where $V_{\mathrm{ext}}$ is a finite sum of sets $V_{i}$, each covered by a single coordinate 
patch $V_{i} \supset\left\{x^{\mu}: r \geqq R_{i},\left|x^{0}\right| \leqq \mathscr{C}_{i}^{1} r^{1-\alpha}+\mathscr{C}_{i}^{2}\right\}, 0 \leqq \alpha<1, \mathscr{C}_{i}^{1}, \mathscr{C}_{i}^{2}, R_{i} \in \mathbb{R}^{+}, r^{\alpha-1}$ is understood as $\ln r$ if $\alpha=1$. Suppose that in $V_{i}$ we have

$$
\begin{gathered}
\Sigma\left|g_{\mu \nu}-\eta_{\mu \nu}\right| r^{\alpha}+\Sigma\left|g_{\mu v, \sigma}\right| r^{\alpha+1}+\Sigma\left|g_{\mu v, \rho \sigma}\right| r^{\alpha+2}+\Sigma\left|g_{\mu v, \rho \sigma \tau}\right| r^{\alpha+3} \leqq c_{1}, \\
\alpha>1 / 2, \quad c_{1} \in \mathbb{R}^{+}
\end{gathered}
$$

and let $g_{\mu v} \in C^{3}\left(V_{\mathrm{int}}\right)$. Let $t$ be a smooth function on $V$, which coincides with $x^{0}$ in each of the ends $V_{i}$, with $\nabla t$ time-like, and suppose that

$$
\|\operatorname{Ricc}\|_{C^{0}}+\|\log a\|_{C^{1}}+\|\nabla T\|_{C^{1}} \leqq C_{1},
$$

where $a^{-2}=-g^{\mu v} t_{, \mu} t_{, v}, T=-a \nabla t$, and the $C^{k}$ norm $\|\cdot\|_{C^{k}}$ is taken with respect to the positive definite metric $g^{\mu v}+2 T^{\mu} T^{v}$ (cf. [Ba1] for details). Suppose that there exist $\varepsilon, C_{1}>0$ such that

$$
\left.\forall i, \quad \forall(t, x) \in V_{i}, \quad \mid(\|\nabla t\| K)(t, x)-\|\nabla t\| K\right)(0, x) \mid \leqq C_{1} r^{-2-\varepsilon},
$$

where $K$ is the trace of the extrinsic curvature of the slices $x^{0}=$ constant. Assume the "bounded interior geometry" condition:

$$
\forall q \in \partial V_{i}, \quad \forall p \in V_{\text {int }} \text { such that } r(q)=R_{i}, p \notin I(q),
$$

we have

$$
|t(p)-t(q)| \leqq C_{2}
$$

$(I(q)$ is the set of points causally related to $q)$ and let

$$
V_{T}=\left\{p \in V_{\text {int }}:|t(p)| \leqq T\right\} \quad \text { be compact for } T=\max _{i}\left(\mathscr{C}_{i}^{1} R_{i}^{1-\alpha}+\mathscr{C}_{i}^{2}\right)+C_{2} .
$$

There exist constants $C^{1}$ and $C^{2}$ depending only upon $R_{i}, c_{1}, C_{1}, C_{2}, \alpha$ and $n$ such that if $\mathscr{C}_{i}^{1} \geqq C^{1}, \mathscr{C}_{i}^{2} \geqq C^{2}$, then there exists in $V$ a complete space-like hypersurface with vanishing mean extrinsic curvature. In the $(x, t)$ coordinates $\Sigma=\operatorname{graph}_{\Sigma_{0}} u=$ $\left\{(x, u): x \in \Sigma_{0}\right\}$, where $\Sigma_{0}=\{q \in V: t(q)=0\}$, with $u$ satisfying the following estimates in each of the asymptotic regions:

$$
\begin{array}{lll}
|u|(1+r)^{\alpha-1}+|D u|(1+r)^{\alpha}+\left|D^{2} u\right|(1+r)^{\alpha+1} \leqq C, & \text { if } & \alpha<1, \\
|u|+|D u|(1+r)+\left|D^{2} u\right|(1+r)^{2} \leqq C(1+\ln (1+r)), & \text { for } & \alpha=1,
\end{array}
$$

with a constant $C$ depending only upon $R_{i}, \mathscr{C}_{i}^{1}, \mathscr{C}_{i}^{2}, c_{1}, C_{1}, C_{2}, \alpha$ and $n$.

Proof. Applying Lemma 2.4 once, as in Theorem 3.1, one obtains a coordinate system $x^{\prime \mu}$, covering in each end at least the set $\Omega_{i}=\left\{r\left(x^{\prime i}\right) \geqq R_{i},\left|t^{\prime}\right| \leqq \min \left(\mathscr{C}_{i}^{1} R_{i}^{1-\alpha}+\mathscr{C}_{i}^{2}\right)\right\}$, in which

$$
\left|K^{\prime}\left(t^{\prime}, x^{\prime}\right)\right| \leqq \mathrm{Cr}^{-2-\varepsilon^{\prime}},
$$

$\varepsilon^{\prime}=\min (2 \alpha-1, \varepsilon)$ with a $\mathscr{C}_{i}^{2}$ independent constant $C$ (cf. Lemma 2.4 point 5), where $K^{\prime}$ is the trace of the extrinsic curvature of the slices $x^{\prime 0}=$ constant. The barrier functions of Proposition 5.1 of [Ba1] can be replaced by the functions $w^{\prime}= \pm \mathrm{Cr}^{-\varepsilon^{\prime}}$, for sufficiently large $C$. The height estimate of Theorem 5.3 of [Ba1] is then obtained using the trial function defined by 


$$
\frac{\Psi^{\prime}}{\Psi}(s)=\left\{\begin{array} { l } 
{ 2 ^ { 1 + \alpha } C _ { 9 } ( m + 1 ) ^ { - 1 - \alpha } , } \\
{ 2 ^ { 1 + \alpha } C _ { 9 } ( m + 2 - s ) ^ { - 1 - \alpha } , } \\
{ C _ { 9 } , }
\end{array} \left\{\begin{array}{l}
0<s \leqq 1 \\
1 \leqq s \leqq m, \\
m \leqq s,
\end{array}\right.\right.
$$

$\Psi(0)=0$. The remaining arguments of $[\mathrm{Ba} 1]$ go through without modifications.

We will call the topology of a three-manifold $\Sigma$ generic if there exists no metric on $\Sigma$ with non-negative scalar curvature (cf. [Wi] and references therein).

Corollary 4.2. Let $(M, g)$ be a globally hyperbolic space-time, $M=\mathbb{R} \times \Sigma$, and suppose that $\Sigma$ has generic topology. If the Einstein tensor satisfies the weak energy condition $\left(G_{\mu \nu} X^{\mu} X^{\nu} \geqq 0\right.$ for all time-like $\left.X^{\mu}\right)$ there exists no time-function on $M$ such that the hypotheses of Theorem 4.1 hold.

Remark. This corollary can be thought of as some sort of a singularity theorem. The results of Schoen and Yau [SY] and the Penrose singularity theorem [HE] imply that Cauchy data for the Einstein equations on a generic manifold $\Sigma$ must develop a singularity either to the future or to the past if the time-like convergence condition holds ${ }^{3}$. The hypotheses of Corollary 4.2 have a clearer physical interpretation since we require only the weak energy condition. It would be extremely useful to replace the conditions of Theorem 4.1 by some conditions with a more transparent geometrical meaning. It has been suggested $[\mathrm{Ba} 4,5]$ that a suitable geometric condition is the absence of "hidden infinities."

In the remainder of this paper, $\Sigma, \Sigma_{\tau}, \tilde{\Sigma}$, denote complete Riemannian threedimensional $C^{m+1, \lambda}$ manifolds of the form $\Sigma=\Sigma_{\mathrm{int}} \bigcup_{i=1}^{N} \Sigma_{i}, \Sigma_{\mathrm{int}}$ - a compact manifold with boundary, $\Sigma_{i}=\mathbb{R}^{3}-B\left(R_{i}\right)$, and the $\Sigma_{i}$ 's are glued to $\Sigma_{\mathrm{int}}$ along the boundaries. The metric is always assumed to be $C^{m, \lambda}$.

A $C^{m+1, \lambda}$ space-time $(M, g)$ with, locally, a $C^{m, \lambda}$ metric will be called strictly stationary and asymptotically flat if

1. $M \approx \mathbb{R} \times \Sigma$, where the embedded leaves $\Sigma_{t}=\{t\} \times \Sigma$ are complete, connected Riemannian submanifolds of $M$;

2. The vector $\partial / \partial t$ is a (strictly) time-like Killing vector;

3. In local coordinates $\left(t, x^{i}\right) \in \mathbb{R} \times\left(\mathbb{R}^{3}-B\left(R_{i}\right)\right), g_{\mu \nu}-\eta_{\mu \nu} \in C_{-\alpha}^{m, \lambda}\left(\mathbb{R}^{3}-B\left(R_{i}\right)\right), m \geqq 2$, $\alpha>0$.

Proposition 4.3. Let $(M \approx \mathbb{R} \times \Sigma, g)$ be a strictly stationary asymptotically flat space-time. There exists a foliation of $M$ by asymptotically flat maximal hypersurfaces $\tilde{\Sigma}_{\tau}, \tau \in \mathbb{R}, \tilde{\Sigma}_{\tau}$ diffeomorphic to $\Sigma$.

Proof. Rather than proving that Bartnik's interior condition (4.4) holds, we shall show directly that any space-like hypersurface $S \subset \mathbb{R} \times \Sigma$ such that $\partial S \subset \mathbb{R} \times \partial \Sigma_{\text {int }}$ must be uniformly bounded in time; this is the key condition for Theorem 4.1 to hold. Let $(t, p) \in S_{\text {int }} \equiv S \cap V_{\text {int }}, V_{\text {int }} \equiv \mathbb{R} \times \Sigma_{\text {int }}$. By time-likeness of $\partial / \partial t$ the hypersurface $S_{\text {int }}$ is a graph over $\Sigma_{\text {int }}: S_{\text {int }}=\left\{(u(p), p): u(p) \in \mathbb{R}, p \in \Sigma_{\text {int }}\right\}$. By compactness of $\Sigma_{\text {int }}$

3 This is a consequence of the fact $[\mathrm{SY}][\mathrm{Ba} 4]$ that every Cauchy surface in $M$ will have trapped surfaces 
a) there exists a minimizing geodesic $\Gamma \subset \Sigma_{\text {int }}$ for the metric induced by $\gamma_{\mu \nu}$ on $\Sigma_{\text {int }}$, from $p$ to $\partial \Sigma_{\text {int }}$,

b) sup $g_{\mu v} X^{\mu} X^{v}=-a<0$, for some constant $a$, where $X$ is the Killing vector $\partial / \partial t$.

c) $\sup |\beta|=b<\infty$, for some constant $b$, where $\beta$ is the shift vector $\left(\beta_{i}=g_{0 i}\right)$. $p \in \Sigma_{\mathrm{int}}$

Let $s$ be any parameter on $\Gamma: \Gamma=\left\{p(s) \in V_{\text {int }}, s \in\left[s_{0}, s_{1}\right]\right\}$. By space-likeness of $S$, the curve $(t(s), p(s)) \equiv(u(p(s)), p(s))$ is space-like, so that in local coordinates we have,

$$
\left|g_{00}\right|\left(\frac{d t}{d s}\right)^{2} \leqq g_{i j} \frac{d x^{i}}{d s} \frac{d x^{j}}{d s}+2 \beta_{i} \frac{d x^{i}}{d s} \frac{d t}{d s} \leqq\left|\frac{d x}{d s}\right|^{2}+\frac{1}{\varepsilon}|\beta|^{2}\left|\frac{d x}{d s}\right|^{2}+\varepsilon\left(\frac{d t}{d s}\right)^{2}
$$

where we have used $2 y z \leqq \varepsilon y^{2}+(1 / \varepsilon) z^{2}$. Setting $\varepsilon=a / 2$ we get

$$
\left|\frac{d t}{d s}\right| \leqq\left[\frac{2}{a}\left(1+\frac{2 b^{2}}{a}\right)\right]^{1 / 2}\left|\frac{d x}{d s}\right| \text {. }
$$

Integrating along the curve $(t(s), p(s))$ we obtain

$$
\begin{aligned}
t\left(s_{1}\right)-t\left(s_{0}\right) & \leqq \int_{s_{0}}^{s_{1}}\left|\frac{d t}{d s}\right| d s \leqq\left[\frac{2}{a}\left(1+\frac{2 b^{2}}{a}\right)\right]^{1 / 2} \int\left|\frac{d x}{d s}\right| d s \\
& \leqq\left[\frac{2}{a}\left(1+\frac{2 b^{2}}{a}\right)\right]^{1 / 2} d(p, q) \leqq\left[\frac{2}{a}\left(1+\frac{2 b^{2}}{a}\right)\right]^{1 / 2} D
\end{aligned}
$$

where $D=\sup _{p, q \in \Sigma_{\text {int }}} d(p, q)<\infty$. In a similar fashion we can estimate the $t$-difference of any two points of $S \cap \partial V_{\text {int }}$, giving an a priori uniform bound for the $t$-difference $\left|t_{1}-t_{2}\right|$ for any two points $\left(t_{1}, p_{1}\right),\left(t_{2}, p_{2}\right) \in S_{\text {int }}$. This and Theorem 4.1 imply the existence of a maximal slice $\tilde{\Sigma}_{0} \subset M$ if $\alpha>\frac{1}{2}$. If $\alpha \ngtr \frac{1}{2}$ stationarity allows us to iterate Lemma 2.9, as in the proof of Theorem 3.1. The slicing $\tilde{\Sigma}_{\tau}$ is given by $\tilde{\Sigma}_{\tau}=\phi_{\tau}\left(\tilde{\Sigma}_{0}\right)$, where $\phi_{\tau}$ is the one-parameter group of diffeomorphisms generated by $X=\partial / \partial t$.

Corollary 4.4. There exists no globally hyperbolic strictly stationary asymptotically flat solution of the Einstein equations with sources satisfying the weak energy condition and a Cauchy surface having generic topology.

\section{Uniqueness}

Throughout this section $\Omega_{\theta, R, T}$ denotes a "boost type" domain:

$$
\Omega_{\theta, R, T}=\left\{x^{\mu}: r(x) \geqq R,\left|x^{0}\right| \leqq r(x)+T\right\} .
$$

We shall write $\Omega_{\theta, R, T}^{x}$ and $\Omega_{\theta, R, T}^{y}$ to denote boost-type domains in different coordinate systems $x$ and $y$ if confusion is likely to occur. In this section we shall only consider maximal slices which are asymptotically flat in a strong sense (cf. Definition 5.1). By doing this we leave aside the interesting question, are all maximal hypersurfaces (say Cauchy surfaces of an asymptotically flat global space-time) asymptotically flat? We adopt the pragmatic point of view that only 
the asymptotically flat maximal slices are relevant for physical application (e.g., see $[\mathrm{An}])$.

Definition 5.1. A space-like hypersurface $\Sigma=\Sigma_{\text {int }} \bigcup_{i=1}^{N} \Sigma_{i}$ of $M$ will be called strongly asymptotically flat if there exist neighborhoods $O_{i}$ of the ends $\Sigma_{i}$ each covered by a single coordinate system $\left(t, x^{i}\right) \in \Omega_{i}=\Omega_{\theta_{i}, R_{1}, T_{1}}=\left\{x^{\mu}: r \geqq R_{i},\left|x^{0}\right| \leqq \theta_{i} r(x)+T_{i}\right\}$ such that

$$
g_{\mu \nu}-\eta_{\mu \nu} \in \mathscr{C}_{-\alpha}^{-m, \lambda}\left(\Omega_{i}\right), \quad \alpha>0
$$

and $\Sigma_{i}=\left\{x^{\mu}: x^{0}=0\right\}$.

Let us recall the following:

Proposition 5.2 [Ch1]. Let $\Sigma$ be a strongly asymptotically flat hypersurface in $M$, let $O \subset M$ be covered by a single coordinate system $y^{\mu} \in \Omega \supset \Omega_{\theta, R, T}^{y}$, with $g_{\mu \nu}-\eta_{\mu \nu} \in \mathscr{C}_{-\alpha}^{m}(\Omega), m \geqq 1,0<\alpha \leqq 1$. Suppose $\Sigma \subset \Omega_{\theta, R, T}^{v}$, let $x^{\mu}$ be the coordinates of Definition 5.1. There exists a Lorentz transformation $\Lambda_{v}^{\mu}(\Sigma) \in O(1, n-1)$ such that

$$
\begin{array}{lll}
y^{\mu}=\Lambda_{v}^{\mu} x^{v}+\zeta^{\mu}(x), & \zeta^{\mu} \in \mathscr{C}_{1-\alpha}^{m+1}\left(\Omega_{\theta, R, T}^{x}\right) & \text { if } \quad \alpha<1, \\
(1+\ln (1+r))^{-1} \zeta^{\mu} \in \mathscr{C}_{0}^{m+1}\left(\Omega_{\theta, R, T}^{x}\right) & \text { if } \quad \alpha=1 .
\end{array}
$$

Remark. If the vacuum Einstein equations are satisfied and we do not artificially restrict the size of $M$, a hypersurface which is asymptotically flat in the sense of Theorem 3.5, is strongly asymptotically flat (cf. the proof of Theorem 3.5).

Proposition 5.3. Let $\Sigma, \tilde{\Sigma}$ be two maximal strongly asymptotically flat hypersurfaces and let $\Sigma, \tilde{\Sigma} \subset \Omega_{\theta, R, T}$ as in Proposition 5.2. If $\Lambda_{v}^{\mu}(\Sigma)=\Lambda_{v}^{\mu}(\tilde{\Sigma})$, then

$$
\begin{array}{ll}
\zeta^{\mu}(\Sigma)-\zeta^{\mu}(\tilde{\Sigma})=\zeta_{0}^{\mu}+\zeta_{1}^{\mu} & \\
\zeta_{0, \nu}^{\mu}=0 \quad \zeta_{1}^{\mu} \in \mathscr{C}_{-\alpha}^{m+1}, & \text { if } \quad \alpha<1, \\
(1+\ln (1+r))^{-1} \zeta_{1}^{\mu} \in \mathscr{C}_{-\alpha}^{m+1}, & \text { if } \quad \alpha=1 .
\end{array}
$$

Proof. Let $\alpha<1$ and let $x_{1}^{\mu}, x_{2}^{\mu}$ be the coordinates of Definition 5.1 for $\Sigma$ and $\tilde{\Sigma}$ respectively. A simple Lorentzian geometry exercise shows that $\widetilde{\Sigma}$ must be included in $\Omega_{\theta_{x_{1}}, R_{x}, T_{x}}^{x_{1}}$ (cf. Corollary 3 in [Ch1]) at least for sufficiently large $r\left(x_{2}\right)$. This together with (5.1) implies that $\tilde{\Sigma}$ is a graph over $\Sigma$ given by the equation

$$
x_{1}^{0}=u\left(x_{1}^{i}\right), \quad u \in C_{1-\alpha}^{m+1}\left(\mathbb{R}^{3}-B(R)\right)
$$

for some $R$ large enough. The calculation of Lemma 2.3 gives

$$
0=\left\|\nabla x_{2}^{0}\right\| K_{x_{2}^{0}}=\left\|\nabla x_{1}^{0}\right\| K_{x_{1}^{0}}(u, x)-\Delta_{0} u+O\left(r^{-1-2 \alpha}\right) .
$$

The mean value theorem, together with $K_{x_{1}^{0}}(0, x)=0$, implies $K_{x_{1}^{0}}(u, x)=O\left(r^{-1-2 \alpha}\right)$, therefore by (5.2)

$$
\Delta_{0} u=O\left(r^{-1-2 \alpha}\right)
$$

so that $u=O(1)$ if $\alpha>1 / 2$, or $u=O\left(r^{1-2 \alpha}\right)$ if $\alpha<1 / 2$. Iterating this a sufficient number of times we obtain $u=$ constant $+O\left(r^{-\alpha}\right)$, which is the desired result. When $\alpha=1$ the result is established in a similar way. 
We will derive a uniqueness theorem for maximal foliations as a consequence of a general estimate for maximal surfaces. This depends on some terminology and results from [Ba2]: a hypersurface $\tilde{\Sigma} \subset M$ will be called weakly space-like if it is locally achronal and separating, i.e. for every $p \in \tilde{\Sigma}$ there is a neighborhood $p \in U$ such that $\tilde{\Sigma} \cap U$ is an embedded, achronal, $C^{0,1}$ hypersurface which is closed in $U$ and separates $U=I^{+}(\tilde{\Sigma} \cap U) \cup \Gamma(\tilde{\Sigma} \cap U) \cup(\tilde{\Sigma} \cap U)$ (disjoint union). The boundary of $\widetilde{\Sigma}$ is defined as

$$
\partial \tilde{\Sigma}=\operatorname{closure}(\tilde{\Sigma})-\tilde{\Sigma} .
$$

Let us note that the definition of a hypersurface insures $\tilde{\Sigma}$ contains no boundary points. $\tilde{\Sigma}$ is locally maximal if $\tilde{\Sigma} \cap U$ maximizes induced area measure, and regular if it is locally the graph of a $C^{k, \alpha}$ function, $k \geqq 2$, with time-like normal vector. It is shown in [Ba2] that if $\tilde{\Sigma}$ is locally maximal, then $\tilde{\Sigma}$ is regular, except perhaps on a singular set

$$
\begin{aligned}
\operatorname{sing}(\tilde{\Sigma})= & \{\gamma(t), 0<t<1, \text { where } \gamma:(0,1) \rightarrow \tilde{\Sigma} \text { is a null geodesic, } \\
& \text { such that the "endpoints" } \gamma(0), \gamma(1) \text { are not in } \tilde{\Sigma}\} .
\end{aligned}
$$

(More precisely, the sequences $\{\gamma(t)\}_{t \downarrow 0},\{\gamma(t)\}_{t \uparrow 1}$, do not have accumulation points in $\tilde{\Sigma}$.) In typical situations, $\operatorname{sing}(\widetilde{\Sigma})$ will be empty, for trivial topological reasons. Finally let us recall the definition of the Lorentzian distance functions [HE]; for $p, q \in M, \Sigma \subset M$, define

$$
d(p, q)=\left\{\begin{array}{l}
0, \quad \text { if } q \notin I^{+}(p) \\
\sup _{q \in \Sigma}\{\operatorname{length}(\gamma): \gamma:[0,1] \rightarrow M \text { is future time-like, } \gamma(0)=p, \gamma(1)=q\} \\
\text { otherwise }
\end{array}\right.
$$

and

$$
d_{\Sigma}(p)=\sup _{q \in \Sigma}\{d(p, q), d(q, p)\}
$$

A set $S$ will be called spatially bounded with respect to a space-like hypersurface $\Sigma$ if there exists a compact subset $G \subset \Sigma$ such that

$$
I(S) \cap \Sigma \subset G .
$$

Theorem 5.4. Let $(M, g)$ be a globally hyperbolic $C^{3}$ Lorentzian manifold satisfying the time-like convergence condition, let $\Sigma$ be a regular locally maximal surface in $M$. Suppose $\tilde{\Sigma}$ is a locally maximal, weakly space-like hypersurface with non-empty boundary $\partial \widetilde{\Sigma}$, such that $\widetilde{\Sigma}$ is spatially bounded with respect to $\Sigma$. Further suppose there is a constant $D$ such that

$$
d_{\Sigma}(p) \leqq D \text { for all } p \in \partial \tilde{\Sigma} .
$$

Then, either

(a) $d_{\Sigma}(p) \leqq D$ for all $p \in \tilde{\Sigma}$, or

(b) there is an incomplete, inextendible, length-maximizing, time-like geodesic in $M$, and moreover, there exists no Cauchy surface containing $\widetilde{\Sigma}$.

Remark. In case (b), there is an upper bound on the distance to the singularity, depending only on the geometry of $M$ in a neighborhood of $G \subset \Sigma$. 
Proof. Suppose (a) is not satisfied, let $G \subset \Sigma$ be the compact set such that $(I(\tilde{\Sigma}) \cap \Sigma) \subset G$. We may assume $G$ has smooth boundary in $\Sigma$. Consider the mapping $K: \mathbb{R} \times Y \rightarrow Z$, where

$$
Y \equiv\left\{u \in C^{2, \alpha}(G),\left.u\right|_{\partial G}=0\right\}, \quad X \equiv C^{0, \alpha}(G)
$$

defined by

$$
K(\sigma, u)=\sigma-K(u), \quad \sigma \in \mathbb{R},
$$

where $K(u)$ is the mean curvature function of $\operatorname{graph}_{G}(u)$, in, say, normal coordinates in a neighborhood of $\Sigma$. The linearization of $K$ at $(0,0) \in \mathbb{R} \times Y$ is given by (cf. e.g. $[\mathrm{CB}])$

$$
D_{Y} K(0,0) v=L v \equiv\left(\Delta-|A|^{2}-\operatorname{Ric}(N, N)\right) v, \quad v \in Y=T_{(0,0)} Y .
$$

The time-like convergence condition implies that $L$ is an isomorphism, therefore by the implicit function theorem there is a $\delta_{0}>0$ such that for any $\delta \in\left(-\delta_{0}, \delta_{0}\right)$ there exist functions $u_{+}, u_{-} \in Y$ such that $K\left(u_{ \pm}\right)= \pm \delta$, moreover $d_{\Sigma}(p) \leqq \varepsilon(\delta)$ for all $p \in S_{ \pm}=\operatorname{graph}_{G}\left(u_{ \pm}\right)$, where $\varepsilon(\delta) \rightarrow 0$, as $\delta \rightarrow 0$. Without loss of generality, we may assume (a) fails to the future of $\Sigma$, and restrict attention to $S=S_{+}$. Since (a) fails, defining

$$
D_{1} \equiv \sup _{p \in \tilde{\Sigma}} d_{\Sigma}(p)
$$

we have $D<D_{1} \leqq \infty$. Choose $\delta$ small enough that $\varepsilon(\delta)<\min \left(1,\left(D_{1}-D\right) / 2\right)$. Direct estimation using the Lorentzian triangle inequality shows that

$$
\left|d_{S}(p)-d_{\Sigma}(p)\right| \leqq \varepsilon \text { for all } p \in \tilde{\Sigma} .
$$

Hence $\left.d_{S}\right|_{\tilde{\Sigma}}$ also does not attain its maximum on $\partial \tilde{\Sigma}$. Since (a) fails, there is a sequence $\left\{p_{k}\right\}_{1}^{\infty}$ in $\tilde{\Sigma}$ such that

$$
D+\varepsilon \leqq d_{S}\left(p_{k}\right) \rightarrow \sup _{p \in \tilde{\Sigma}} d_{S}(p) .
$$

Suppose $\left\{p_{k}\right\}$ has an accumulation point $p \in \tilde{\Sigma} \cup \partial \tilde{\Sigma}$. (Recall that $p$ is an accumulation point of the sequence $\left\{p_{k}\right\}_{1}^{\infty}$ if, for every (open) neighborhood $\mathscr{U} \ni p$, and positive integer $N$, there is $k \geqq N$ such that $p_{k} \in \mathscr{U}$ ). By passing to a subsequence we may assume $p_{k} \rightarrow p \in \tilde{\Sigma}$ since, by construction, $p \notin \partial \tilde{\Sigma}$. Since $I(\tilde{\Sigma}) \subset D(G)=D(S)$, we see that $\tilde{\Sigma}$ is spatially bounded with respect to $S$, so there is a time-like geodesic $\gamma$ which realises $d_{S}(\widetilde{\Sigma})$, and $\gamma$ has endpoint $\gamma(d)=p$. Since $p$ maximizes $d_{S}(\cdot)$ on $\widetilde{\Sigma}, p$ cannot lie in a null geodesic in $\tilde{\Sigma}$ and thus by the regularity of variational maximal surfaces [Ba2], $\tilde{\Sigma}$ is regular in a neighborhood of $p$. However, the second variation inequality for time-like geodesics from $S$ to $\tilde{\Sigma}$ [HE], [BF] shows

$$
\begin{aligned}
0 & \geqq \int_{0}^{d} \operatorname{Ric}\left(\gamma^{\prime}, \gamma^{\prime}\right) d s+K_{S}(\gamma(0))-K_{\tilde{\Sigma}}(p) \\
& \geqq \int_{0}^{d} \operatorname{Ric}\left(\gamma^{\prime}, \gamma^{\prime}\right) d s+\delta,
\end{aligned}
$$

which is incompatible with the time-like convergence condition and hence $\left\{p_{k}\right\}$ cannot have an accumulation point in $\widetilde{\Sigma} \cup \partial \widetilde{\Sigma}$. The definition of $\partial \widetilde{\Sigma}$ then implies 
$\left\{p_{k}\right\}$ cannot accumulate in $M$ either, and to complete our proof we have to show existence of an inextendible incomplete geodesic in $M$. Let $\left\{q_{k}\right\}$ be a sequence in $S$ such that

$$
d_{S}\left(p_{k}\right)=d\left(q_{k}, p_{k}\right),
$$

and let $\gamma_{k}$ be the time-like geodesic realizing $d\left(q_{k}, p_{k}\right)$. Since $\tilde{\Sigma}$ is spatially bounded, we may assume $\left\{q_{k}\right\}$ has a limit point $q \in S$, and then $\gamma_{k} \rightarrow \gamma$, where $\gamma$ is the future time-like geodesic normal to $S$ through $q$. Since $\gamma$ is the limit of length-maximising geodesics, it is itself length-maximizing amongst curves meeting $S$. In particular, the Focussing Lemma [HE] implies $\gamma$ has length $\leqq 1 / \delta<\infty$ and $\gamma$ is inextendible in $M$. Since $\gamma$ does not meet $\tilde{\Sigma}$, and every point of $\gamma$ has some points of the sequence $\left\{p_{k}\right\} \subset \widetilde{\Sigma}$ in its future, we see that $\widetilde{\Sigma}$ cannot lie in any Cauchy surface of M. q.e.d.

The following example illustrates case (b) of the theorem. Let

$$
\begin{aligned}
M & =\left\{(x, t) \in \mathbb{R}^{3,1}, t<r(x)+1\right\} ; \\
\Sigma & =\left\{(x, 0) \in \mathbb{R}^{3,1}, x \in \mathbb{R}^{3}\right\} ; \\
\tilde{\Sigma} & =\operatorname{graph}(u), u: \mathbb{R}^{3}-\{0\} \rightarrow \mathbb{R},
\end{aligned}
$$

where $u(x)$ is the radially symmetric solution of $K(u)=0$ such that $u(0)=1$ and $u(x) \rightarrow 0$ as $r(x) \rightarrow \infty$ [BS]. The submanifold $\tilde{\Sigma}_{100}=\tilde{\Sigma} \cap\{(x, t): r(x) \leqq 100\}$ is spatially bounded and close to $\Sigma$ on $\partial \tilde{\Sigma}_{100}$, and $d_{\Sigma}$ attains its supremum on $\widetilde{\Sigma}_{100}$ at the "missing point" $(0,1) \in \mathbb{R}^{3,1}$. The time-like geodesic $\gamma: t \mapsto(0, t) \in \mathbb{R}^{3,1}$, $0 \leqq t<1$, is inextendible in $M$.

Let $\left\{y_{i}^{\mu}\right\}$ denote a collection of coordinate systems, one in each of the asymptotic four-ends of $M$, each covering at least some boost-type domain $\Omega_{\theta_{i}, R_{i}, T_{l}}^{y_{i}}$ with $\left(g_{\mu \nu}-\eta_{\mu \nu}\right) \in C_{-\alpha}^{2}\left(\Omega_{\theta_{i}, R_{i}, T_{i}}^{y_{i}}\right)$. We shall say that a foliation $\Sigma_{\tau}$ is included in a boost-type domain of the coordinates $\left\{y_{i}^{\mu}\right\}$ if for every asymptotic three-end $\left(\Sigma_{\tau}\right)_{i}$ there exists $\theta_{i}(\tau), R_{i}(\tau), T_{i}(\tau)$ such that $\left(\Sigma_{\tau}\right)_{i} \subset \Omega_{\theta_{i}(\tau), R_{i}(\tau), T_{i}(\tau)}^{y_{i}}$. Using Theorem 5.4 we show: ${ }^{4}$

Theorem 5.5. Let $(M, g)$ be a $C^{3}$ globally hyperbolic Lorentz manifold satisfying the time-like convergence condition. Suppose $\Sigma_{\tau_{1}}^{1}, \Sigma_{\tau_{2}}^{2}$ are strongly asymptotically flat maximal foliations in $M$, let $\Sigma_{0}^{1}$ be a Cauchy surface in $M$, suppose that the foliations $\Sigma_{\tau_{1}}^{1}, \Sigma_{\tau_{2}}^{2}$ are included in a boost-type domain of some set of coordinates $\left\{y_{i}^{\mu}\right\}$. If $\Lambda_{v}^{\mu}\left(\Sigma_{\tau_{1}}^{1}\right)=\Lambda_{v}^{\mu}\left(\Sigma_{\tau_{2}}^{2}\right)$, where $\Lambda_{v}^{\mu}\left(\Sigma_{\tau_{a}}^{a}\right), a=1,2$, are given by Proposition 5.2, then either

a) the foliations $\Sigma_{\tau_{1}}^{1}, \Sigma_{\tau_{2}}^{2}$ coincide, or

b) $M$ is time-like geodesically incomplete and the slices $\Sigma_{\tau_{2}}^{2}$ are not contained in any Cauchy surface.

Proof. By Proposition 5.3, for every hypersurface $\tilde{\Sigma}=\Sigma_{c_{2}}^{2} \subset \Omega_{\theta, R, T}^{y}$ there exists $c_{1} \in R$ such that $\Sigma=\Sigma_{c_{1}}^{1}$ satisfies $d_{\Sigma}(\{p \in \Sigma, r(p) \geqq R\}) \rightarrow 0$ as $R \rightarrow \infty$. Applying Theorem 5.4 to $\tilde{\Sigma} \cap\{p \in M: r(p) \leqq R\}=\tilde{\Sigma}_{R}$ and letting $R \rightarrow \infty$ shows that either

\footnotetext{
4 A uniqueness theorem without the hypothesis that the asymptotically flat coordinates on the maximal slices can be extended to a boost type domain can be obtained using Proposition 3.1.4 of [Ch2] instead of Proposition 5.2
} 
$\Sigma_{c_{1}}^{1}=\Sigma_{c_{2}}^{2}$ or $\Sigma_{c_{2}}^{2}$ is not contained in any Cauchy surface, and $M$ is time-like geodesically incomplete. Since this holds for any slice $\Sigma_{\tau_{2}}^{2} \subset \Omega_{\theta, R, T}^{y}$, the result follows.

Theorem 5.5 shows that complete maximal Cauchy hypersurfaces can be used to eliminate "time supertranslations." It should however be stressed that the freedom of performing supertranslations on each individual maximal slice still remains.

Acknowledgements. P.T.C. and N.Ō.M. are grateful to the Centre for Mathematical Analysis, Australian National University, for friendly hospitality during part of the work of this paper.

\section{References}

[An] Andersson, L.: Geom and Phys. 4, 289-314 (1988)

[As] Ashtekar, A.: Found of Phys. 15, 419-431 (1984)

[Ba1] Bartnik, R.: Commun. Math. Phys. 94, 155-175 (1984)

[Ba2] Bartnik, R.: Acta Math. 161, 145-181 (1988)

[Ba3] Bartnik, R.: Commun. Pure App. Math. 39, 669-693 (1986)

[Ba4] Bartnik, R.: Proc of the 5th Marcel Grossman Meeting. Blair, D. (ed.) (in press)

[Ba5] Bartnik, R.: Commun. Math. Phys. 117, 615-624 (1988)

[BF] Brill, D., Flaherty, F.: Commun. Math. Phys. 50, 157-165 (1976)

[BOM] Beig, R., Ō Murchadha, N.: Ann. Phys. 174, 463-498 (1987)

[Br] Brill, D.: Proc. of the Third Marcel Grossman Meeting. Ning, H. (ed.). Amsterdam: North-Holland 1982

[BS] Bartnik, R., Simon, L.: Commun. Math. Phys. 87, 131-152 (1982)

[CB] Choquet-Bruhat, Y.: Ann. di Scuola Norm. Sup. Pisa 3, 361-376 (1976)

[CBY] Choquet-Bruhat, Y., York, J. W.: In General relativity and gravitation. Held, A. (ed.). New York: Plenum Press 1980

[Ch1] Chruściel, P. T.: Commun. Math. Phys. 120, 233-248 (1988)

[Ch2] Chruściel, P. T.: In Topological properties and global structure of space-time. Bergmann, P. G., de Sabbata, V. (eds.), pp. 49-59. New York: Plenum Press 1986

[Ch3] Chruściel, P. T.: On the energy of the gravitational field at spatial infinity. In: Conference on Mathematical Relativity, Canberra 1988. Proceeding of the CMA Vol. 19, Bartnik, R. (ed.)

[COM] Christodoulou, D., Ō Murchadha, N.: Commun, Math. Phys. 80, 271-300 (1981)

[CSCB] Chaljub-Simon, A., Choquet-Bruhat, Y.: Ann. Fac. Sci. Toulouse 1, 9-25 (1979)

[GT] Gilbarg, D., Trudinger, N.: Elliptic partial differential equations of second order. Berlin, Heidelberg, New York: Springer 1983

[HE] Hawking, S., Ellis, G. F. R.: The large scale structure of space-time. Cambridge: Cambridge University Press 1973

[Me] Meyers, N.: J. Math. Mech. 12, 247-264 (1963)

[OM] Ō Murchadha, N.: J. Math. Phys. 27, 2111-2128 (1986)

[ST] Schoen, R., Yau, S. T.: Commun. Math. Phys. 79, 231-260 (1981)

[Wi] Witt, D. M.: Phys. Rev. Lett. 57, 1386-1389 (1986)

Communicated by S.-T. Yau

Received June 5, 1989 
